\title{
A enfermagem frente às manifestações clínicas da depressão em idosos que sofreram abandono: revisão integrativa da literatura
}

\author{
Nursing facing the clinical manifestations of depression in the elderly who were \\ abandoned: integrative literature review
}

Enfermería frente a manifestaciones clínicas de depresión en ancianos abandonados: revisión integral de literatura

Yasmin Beatriz Reis da Silva Lima ${ }^{1 *}$, Ayanne Castro de Miranda1, Eduardo Soares Jucá1, Stéphanie Caroline Vaz Dias ${ }^{1}$, Suellen Karoline de Lima Pinheiro ${ }^{1}$, Sara do Socorro da Silva Silva ${ }^{1}$, Ana Paula Moia Rodrigues Viana ${ }^{1}$, Paulo Gabriel Moreira Portilho', Melissa Barbosa Martins ${ }^{1}$, Viviane Ferraz Ferreira de Aguiar ${ }^{1}$.

\section{RESUMO}

Objetivo: Mapear o processo de enfermagem, com ênfase nas intervenções de enfermagem, do cuidado ao idoso em contexto de abandono e acometido a depressão. Métodos: Este estudo consiste numa Revisão Integrativa da Literatura. Foi realizada pesquisa nas bases de dados Scientific Electronic Library Online (SciELO) e Literatura Latino-Americana e do Caribe em Ciências da Saúde (Lilacs), utilizando-se a combinação de descritores em idioma português segundo consulta aos Descritores em Ciências da Saúde (DeCS). Para apoiar a análise dos dados, foi utilizado o software IRAMUTEQ e para o processamento de dados utilizou-se a Classificação Hierárquica Descendente (CHD). Resultados: A qualidade de vida não está restrita somente ao físico, mas também ao social. Verificou-se a prevalência de sintomas depressivos em idosos que foram institucionalizados e com isso perderam a convivência com a comunidade. Dessa forma o abandono favorece a aquisição de sintomas depressivos no idoso, por conta do sentimento de solidão. Considerações finais: Há poucas referências correlacionando o processo de enfermagem com a população idosa acometida de quadros de transtorno psicológico. Sendo necessário o desenvolvimento de estudos visando promover intervenções de enfermagem ao cuidado do idoso.

Palavras-chave: Idoso, Maus-tratos ao idoso, Depressão, Enfermagem, Processo de enfermagem.

\begin{abstract}
Objective: Map the nursing process, with an emphasis on nursing interventions, care for the elderly in the context of abandonment and affected by depression. Methods: The study consists of an Integrative review of the literature. We researched the databases SciELO and Lilacs, using a combination of the descriptors in Portuguese according to a search in Descritores em Ciências da Saúde (DeCS). To support data analysis, the IRAMUTEQ software was used, and for data processing, we used the Descending Hierarchical Classification (HDC). Results: Most articles point out that the quality of life is not restricted only to the physical, but also to the social. There was a prevalence of depressive symptoms in elderly people who were institutionalized and, as a result, lost contact with their communities. Thus, abandonment favors the acquisition of depressive symptoms in the elderly, due to the feeling of loneliness. Final considerations: There are few references correlating the nursing process with the elderly population with psychological disorders. It is necessary to develop studies to promote nursing interventions to care for the elderly.
\end{abstract}

Keywords: Elderly, Elderly abuse, Depression, Nursing, Nursing process.

1 Universidade Federal do Pará (UFPA), Belém - PA. *E-mail: yasminb539@gmail.com 


\section{RESUMEN}

Objetivo: Mapear el proceso de enfermería, con énfasis en las intervenciones de enfermería, el cuidado de los ancianos en el contexto del abandono y la depresión. Métodos: Este estudio consiste en una Revisión integradora de la literatura. Se realizó una búsqueda en las bases de datos SciELO y Lilacs, utilizando la combinación de descriptores en portugués de acuerdo con la base Descritores de Ciências da Saúde (DeCS). Para apoyar el análisis de datos, se utilizó el software IRAMUTEQ y para el procesamiento de datos se usó la Clasificación Jerárquica Descendiente (CJD). Resultados: Se observó que la mayoría de los artículos señalan que la calidad de vida no se restringe solamente a lo físico, si no también a lo social. Hubo una prevalencia de síntomas depresivos en las personas mayores que se institucionalizaron y, como resultado, perdieron contacto con la comunidad. De esta manera el abandono favorece la aparición de síntomas depresivos en el adulto mayor, debido a la sensación de soledad. Consideraciones finales: Existen pocas referencias que correlacionen el proceso de enfermería con la población de edad avanzada con trastornos psicológicos. Es necesario desarrollar estudios para promover intervenciones de enfermería en el cuidado de los ancianos.

Palabras clave: Ancianos, Abuso de ancianos, Depresión, Enfermería, Proceso de enfermería.

\section{INTRODUÇÃO}

O envelhecimento é processo biológico inevitável e uma realidade pertinente nas sociedades atuais. Esse fenômeno demográfico é causado, principalmente, pela diminuição das taxas de natalidade e o aumento da expectativa de vida da população. Porém, é necessário que a longevidade e a qualidade de vida estejam associadas, pois, o envelhecimento também é marcado pela diminuição das habilidades motoras e funcionais, e também pelo aparecimento de doenças. Portanto, para um envelhecimento bem sucedido, é importante que os idosos recebam apoio da sociedade, a fim de adquirir um bem estar físico, mental e social (LOBO AJS, et al., 2014).

Verifica-se no Brasil um número crescente de pessoas que possuem mais de 60 anos de idade. Neste contexto, cuidar dessa população é um grande desafio, haja vista que, uma parcela significativa de idosos possuem baixos níveis econômicos e maior ocorrência de doenças crônicas, desencadeando doenças psíquicas, como a depressão (BEZERRA FC, et al., 2012).

A depressão consiste-se em uma enfermidade mental onde o indivíduo apresenta sofrimento psíquico. Em idosos a depressão é caracterizada como uma síndrome com inúmeros aspectos clínicos, em que, cerca de 2 a $14 \%$ desses idosos que vivem na comunidade apresentam depressão, e $30 \%$ dos que moram em instituições. Porém, por razões socioculturais, uma parcela deles resistem ou omitem os sintomas mentais como exemplo disforia, queixas somáticas, hipocondria, baixo autoestima e entre outros (FLORINDO $S$, et al., 2002).

Dentro desse contexto, percebe-se que as causas da depressão em idosos relacionam-se a um conjunto de fatores sociais, neurobiológicos e entre outros. A patologia pode ser manifestada em momentos de perda de qualidade de vida, associada a doenças clínicas graves, luto e abandono. Ademais, os pacientes idosos deprimidos possuem uma maior chance de cometer suicídio do que os não deprimidos, tendo isso em vista, é importante que se tenha um tratamento com os profissionais da saúde, a fim de reduzir o sofrimento psíquico (FLORINDO S, et al., 2002).

Cabe ressaltar, que muitas famílias têm a percepção que os idosos estão inaptos a exercerem qualquer atividade, com isso, muitos deles são levados às instituições asilares. Diante disso, percebe-se, uma parcela de idosos com sentimentos de abandono, solidão, mágoa e/ou como um peso para a família. A institucionalização provoca nos idosos a sensação de perda do papel e da participação social, além disso, os mesmos sentem arrependimento por não terem desfrutado a vida. Tais sentimentos tornam o idoso infeliz nos estágios finais da vida, podendo desencadear possíveis alterações neurológicas como a depressão (EVANGELISTA RA, et al., 2014). 
Portanto, o cuidado e a assistência de enfermagem são analisados a partir do bem-estar vivenciado pelo idoso, visando o atendimento de suas necessidades. Ao evidenciar o idoso como sendo sujeito participante da atenção da enfermagem, considera-se este incorporado na sociedade e em grupos sociais (SILVA PLN, et al., 2014). Ademais, é dever da enfermagem estar atenta para o universo do outro e ter em vista as concepções sobre o que o cliente avalia e sente, como sendo necessidade de saúde. Assim, as intervenções de enfermagem são ações práticas que podem ultrapassar o aspecto biológico de acordo com a demanda da clientela (SILVA EP, et al., 2019.)

Diante disso, este estudo se justifica pela necessidade da busca a percepção de como o abandono a pessoa idosa influencia a um quadro depressivo. Ademais, o presente estudo teve como objetivo mapear o processo de enfermagem, com ênfase nas intervenções de enfermagem, do cuidado ao idoso em contexto de abandono e acometido a depressão e descrever quais aspectos são ressaltados nos artigos em relação a esse tema.

\section{MÉTODOS}

O presente estudo consistiu em uma Revisão Integrativa da Literatura (RIL), para a elaborar a revisão integrativa foram necessários seis etapas, a primeira etapa foi a elaboração da pergunta norteadora, a segunda foi busca ou amostragem na literatura, a terceira etapa foi a coleta de dados, a quarta etapa foi análise crítica dos estudos incluídos, conseguinte, a quinta etapa foi discussão dos resultados e por fim, a sexta etapa ao qual foi apresentação da revisão integrativa ao qual os dados são interpretados, sintetizados e conclusões são formuladas originadas dos vários estudos incluídos na revisão integrativa (MENDES KDS, et al., 2008).

Foi realizada pesquisa nas bases de dados Scientific Electronic Library Online (SciELO) e Literatura Latinoamericana e do Caribe em Ciências da Saúde (Lilacs), utilizando-se a combinação de descritores em idioma português segundo consulta aos Descritores em Ciências da Saúde (DeCS). Na realização das buscas foram feitas seis combinações dos termos, sendo: Enfermagem AND Depressão AND Idoso; Enfermagem AND Maus-tratos ao Idoso AND Idoso; Processo de Enfermagem AND Idoso; Processo de Enfermagem AND Depressão. As bases acima foram delimitadas a partir da facilidade de acesso aos trabalhos que, em sua maioria estão disponíveis em sua forma completa.

A busca dos estudos deu-se a partir de alguns critérios de inclusão, sendo eles: a) artigos escritos no idioma português e inglês; e b) artigos que abordassem a problemática do abandono ao idoso, fazendo correlação a Enfermagem e as suas intervenções; c) artigos produzidos pela enfermagem. Os estudos que não cediam o artigo em sua forma completa, os estudos repetidos, teses, dissertações, monografias, relatórios, entre outros, não foram inseridos na pesquisa. Elegeu-se por limitar o período de publicação, o qual foi 2014 a 2018. A pesquisa nas bases de dados ocorreu em outubro de 2019.

Para a seleção dos artigos, os mesmos foram analisados de forma livre pelos autores, com base em seus resumos, e posteriormente, na leitura completa destes quando o primeiro recurso era insuficiente para determinar sua seleção. As eliminações de artigos, foram fundamentadas e as divergências resolvidas em consenso. Estabelecida a seleção dos artigos, eles foram analisados em sua totalidade e submetidos a duas etapas de análise (quantitativa e qualitativa). Num primeiro momento foi feita uma análise quantitativa, considerando: a) o ano de publicação e sua quantidade; b) o limiar da publicação; c) a metodologia utilizada; d) o idioma que foi publicado e) a área temática da publicação. Realizando-se essa fase, seguiu-se com a análise qualitativa dos artigos selecionados e a análise feita pelo software Interface de $\mathrm{R}$ pour les Analyses Multidimensionnelles de Textes et de Questionnaires (IRAMUTEQ).

Para apoiar a análise dos dados desta pesquisa, foi utilizado o software IRAMUTEQ. O programa permite diferentes processamentos e análises estatísticas de textos produzidos. Dentre as vantagens está a de ser gratuito na lógica de open source - código aberto. É ancorado no software $R$ e na linguagem de programação python. Para a análise, definiu-se o método da Classificação Hierárquica Descendente (CHD), proposto por Reinert, em que os textos são classificados em função de seus respectivos vocabulários e o conjunto deles se divide pela frequência das formas reduzidas (CAMARGO BV e JUSTO AM, 2013). 


\section{RESULTADOS}

Realizou-se a busca dos artigos nos bancos de dados, sendo localizados 7.410 estudos referentes aos descritores utilizados e após a adoção dos critérios de inclusão, selecionou-se 15 estudos para processamento e análise. O Corpus foi constituído por dados existentes nas conclusões dos estudos, colocados em um único arquivo de texto, conforme orientações do tutorial do IRAMUTEQ. (CAMARGO BV e JUSTO AM, 2013). O corpus foi formado pelo conjunto de textos a ser analisado, fragmentado, pelo software, em segmentos de texto (Figura 1).

Figura 1 - Identificação, seleção e inclusão das publicações na amostra da revisão integrativa. Belém - PA, Brasil, 2019.
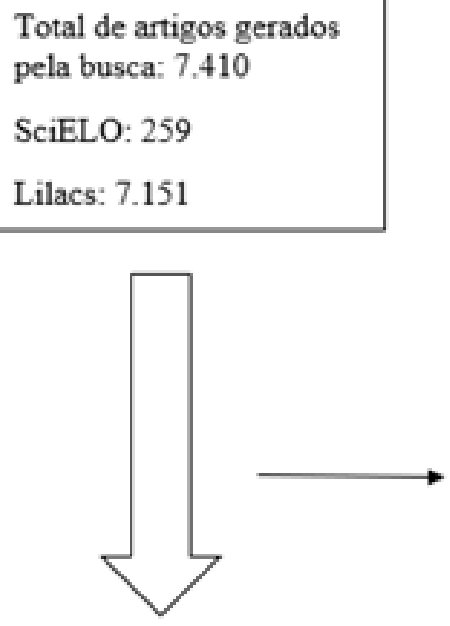

Artigos excluidos: 7.136

- Artigos repetidos: 1

- Gray Literature: 7.066

- Artigos que não fazem menção à

Problemática de Depressão em

idosos que sofreram abandono: 70

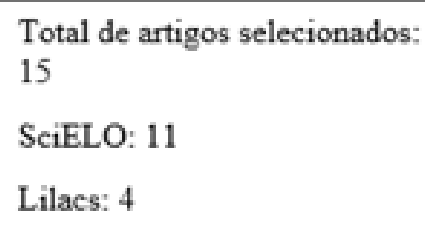

Fonte: Lima YBRS, et al., 2020.

Cabe ressaltar que grande parte dos artigos inseridos na revisão estavam publicados em revistas específicas da área da Enfermagem. Acerca da base de dados, 11 estudos foram encontrados no SciELO e 4 na Lilacs. Os estudos denotados nesses artigos foram elaborados por enfermeiros, abrangendo especialistas, mestres e doutores, e as pesquisas foram produzidas em instituições de longa permanência, hospitais e instituições psiquiátricas.

No que diz respeito a metodologia dos artigos apurados, verificou-se que a maioria é delineado como exploratório-descritivo com abordagem qualitativa; e o restante de caráter exploratório-descritivo com abordagem quantitativa; e a minoria, qualitativo (Quadro 1).

Os referenciais teóricos não foram visíveis na maioria dos estudos. Todavia, foi possível verificar que são abordadas, a atividade técnica, questões humanísticas como integralidade, escuta qualificada e necessidade de interação com o idoso. 
Quadro 1 - Identificação dos artigos selecionados.

\begin{tabular}{|c|c|c|c|c|}
\hline ARTIGO & ANO & TÍTULO DO ARTIGO & PERIÓDICO & AUTORES \\
\hline 1 & 2017 & Cuidado ao paciente idoso hospitalizado: implicações para a equipe de enfermagem & Lilacs & MENEGUIN S, et al. \\
\hline 2 & 2014 & Prevalência de sintomatologia depressiva e fatores associados entre idosos institucionalizados. & SciELO & LEAL MCC, et al. \\
\hline 3 & 2018 & $\begin{array}{l}\text { Vulnerabilidades, depressão e religiosidade em idosos internados em uma unidade de } \\
\text { emergência }\end{array}$ & SciELO & NERY BLS, et al. \\
\hline 4 & 2016 & $\begin{array}{l}\text { Intervenções de Enfermagem para idosos que envelheceram em instituições psiquiátricas: } \\
\text { mapeamento cruzado }\end{array}$ & Lilacs & SILVA T, et al. \\
\hline 5 & 2016 & Prevalência de sintomas depressivos e sinais de demência em idosos na comunidade & Lilacs & LENTSCK MH, et al. \\
\hline 6 & 2014 & $\begin{array}{l}\text { Sintomas depressivos em idosos: comparação entre residentes em condomínio específico para } \\
\text { idoso e na comunidade }\end{array}$ & Lilacs & TESTON EF, et al. \\
\hline 7 & 2014 & Percepções e vivências dos idosos residentes de uma instituição asilar & SciELO & EVANGELISTA RA, et al. \\
\hline 8 & 2018 & $\begin{array}{l}\text { Gerontotecnologias utilizadas pelos familiares/cuidadores de idosos com Alzheimer: } \\
\text { contribuição ao cuidado complexo }\end{array}$ & SciELO & ILHA S, et al. \\
\hline 9 & 2018 & $\begin{array}{l}\text { Stressors factors experienced by hospitalized elderly from the perspective of the Neuman } \\
\text { Systems Model }\end{array}$ & SciELO & ROSA PH, et al. \\
\hline 10 & 2018 & Elderly caring for the elderly: spirituality as tensions relief & SciELO & SILVA MCM, et al. \\
\hline 11 & 2018 & O ensino de enfermagem gerontológica nas instituições públicas brasileiras de ensino superior & SciELO & RODRIGUES RAP, et al. \\
\hline 12 & 2018 & Board games: gerotechnology in nursing care practice & ScIELO & $\begin{array}{l}\text { OLYMPIO PCAP e ALVIM } \\
\text { NAT. }\end{array}$ \\
\hline 13 & 2018 & The meaning of religion/religiosity for the elderly & SciELO & $\begin{array}{l}\text { OLIVEIRA ALB e MENEZES } \\
\text { TMO. }\end{array}$ \\
\hline 14 & 2014 & Idosos residentes em uma instituição de longa permanência: adaptação à luz de Callista Roy & SciELO & FREITAS MC, et al. \\
\hline 15 & 2014 & Qualidade e condições de vida sob a ótica dos residentes de um condomínio do idoso & SciELO & TESTON EF e MARCO SS. \\
\hline
\end{tabular}

Fonte: Lima YBRS, et al., 2020. 
Logo após, realizou-se a identificação juntamente com investigação dos domínios textuais assim como os significados classificando-os com seus respectivos significados em classes: 1- A idade como principal fator de adoecimento; 2- As características sociais e culturais que influenciam no adoecimento; 3-Religiosidade e saúde: atuação ética do enfermeiro, e 4- Prevalência de sintomatologia depressiva e idosos. Mediante a Classificação Hierárquica Descendente (CHD), o IRAMUTEQ apresentou o Diagrama de Similaridade (DI) das classes criadas a partir do corpus inserido no programa.

Para a construção do DI, que ilustra as subdivisões as quais sucederam-se feitas no corpus, até que alcançasse a classificação final. Por fim, para a análise posterior foram consideradas aquelas palavras que obtiveram maior frequência. As classes foram descritas pelas palavras mais significativas e suas respectivas associações com a classe (Figura 2).

Figura 2 - Estrutura temática da depressão em idosos e as intervenções de enfermagem.

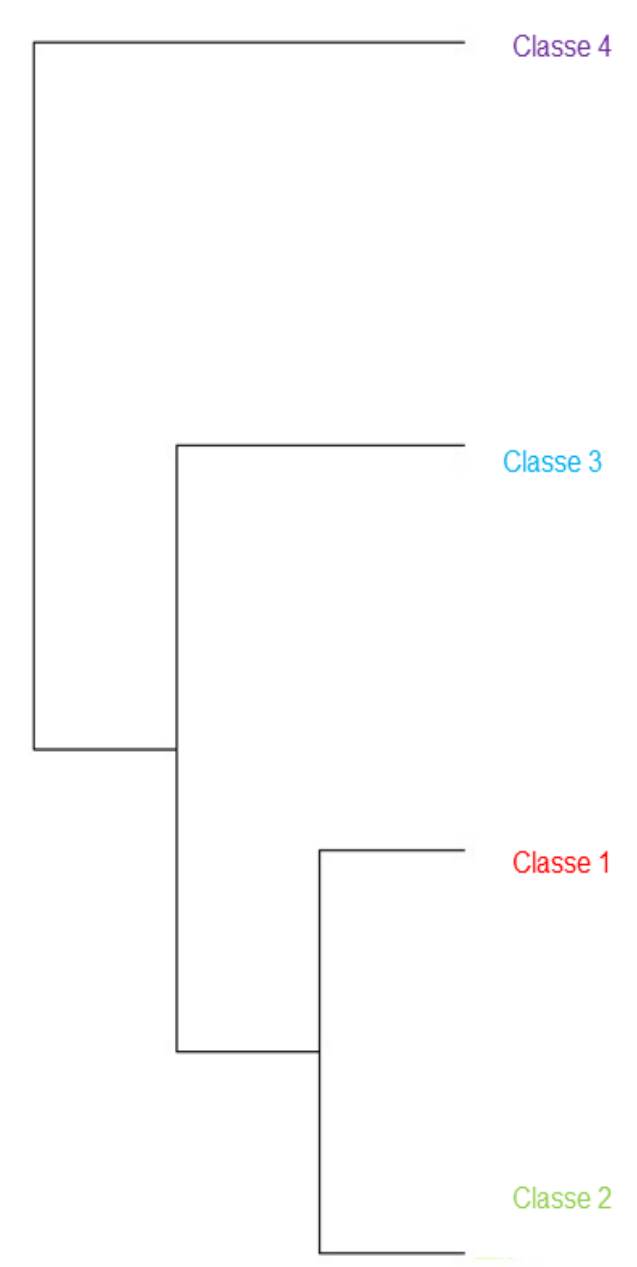

Fonte: Lima YBRS, et al., 2020.

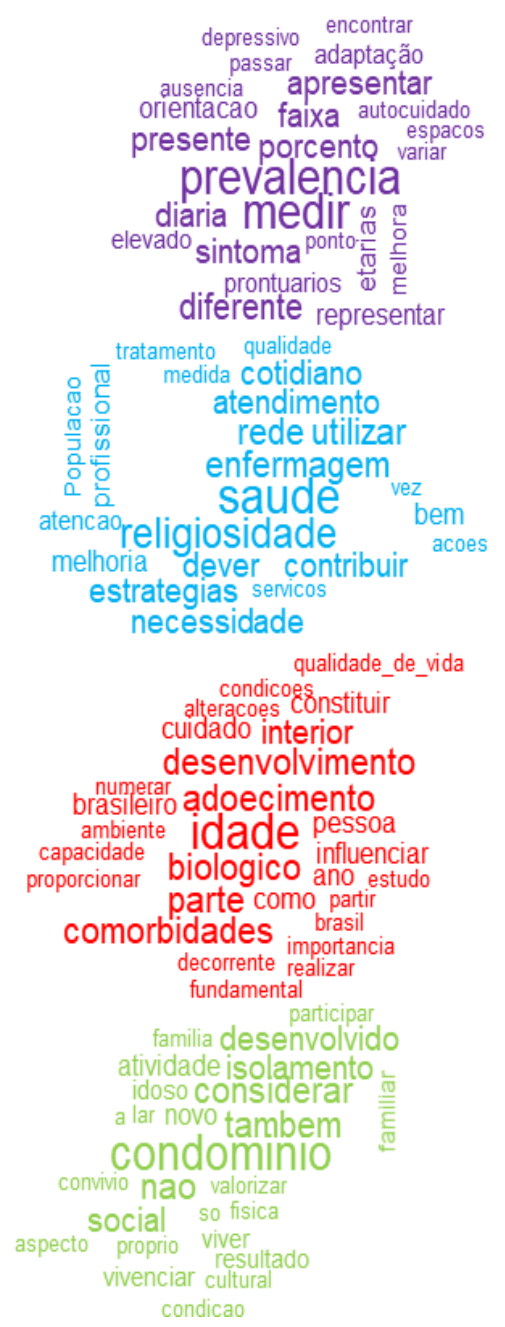

Notou-se que a maioria dos artigos apontam que a qualidade de vida não está restrita somente ao físico, mas também ao social. Tal resultado corrobora que a independência seja um dos fatores primordiais para a melhora da autoestima e qualidade do idoso. Assim, é necessário reconhecer que a individualidade de cada idoso é de suma importância para a valorização de sua saúde e para o planejamento da assistência prestada pelo enfermeiro.

Percebeu-se que há pouquíssimos estudos que abordam as intervenções de enfermagem, assim, é necessário proporcionar o cuidado ao idoso, para que se possa manter sua autonomia, sendo fundamental a assistência de enfermagem individualizada, envolvendo o ambiente familiar, incentivando o desenvolvimento da autossuficiência e estimulando a capacidade de autocuidado dentro das suas limitações humanas. 
Através dos estudos, verificou-se a prevalência de sintomas depressivos em idosos que foram institucionalizados e com isso perderam a convivência com a comunidade. Ademais, constatou-se que os idosos mais vulneráveis ao surgimento de sinais de depressão são os que se encontram na faixa etária de 70 a 80 anos.

Quando feita a avaliação dos estudos, identificou-se que a maioria dos idosos brasileiros que não possuem convívio familiar, tende a desenvolver depressão. Diante disso, nota-se que além do cuidado de enfermagem é de fundamental importância o apoio familiar, com o intuito de melhorar o quadro depressivo, por intermédio, do convívio social e do estímulo de atividades.

O enfermeiro em seu papel, avalia os diferentes aspectos para fomentar o cuidado individualizado aquele idoso em situação de vulnerabilidade. Identificou-se que para promover o cuidado e recuperação do paciente, o profissional de enfermagem deve identificar os problemas relacionados ao quadro depressivo, e em seguida deve planejar as intervenções de enfermagem, como: Promoção do autocuidado, estimular atividades físicas, reduzir o contato do paciente com fatores estressores e quando necessário, possibilitar o acesso à religiosidade, promovendo a saúde espiritual.

É de suma importância inferir que os idosos assim como o resto da população brasileira pode ser diagnosticado com depressão, fator que pode se desenvolver pela perda de autonomia e, na maioria dos casos o isolamento familiar, uma realidade que se perpetua por todo o país.

Dessa forma o abandono favorece a aquisição de sintomas de depressão no idoso, porque o sentimento de solidão pode contribuir para que a saúde desse idoso esteja abalada, na qual a negligência familiar ajuda ainda mais para se adquirir depressão. Com isso, o enfermeiro deve observar a saúde mental desse paciente.

\section{DISCUSSÃO}

Segundo Corrêa ERP e Miranda-Ribeiro A (2017), a expectativa de vida é um indicador de mortalidade sintético. Ela é estimada a partir das condições sociais e ambientais em que uma determinada população vive. Em estudos, verificou-se que a partir da metade do último século, as taxas de natalidade tiveram uma queda mais acentuada do que as taxas de mortalidade. Tudo isso levou a uma diminuição do crescimento populacional, o aumento da expectativa de vida, e consequentemente, o Envelhecimento da população brasileira. (CARMO EH, et al., 2003).

Um fator importante a ser levado em consideração quando se pensa em qualidade de vida dos idosos é a religiosidade individual. Cientistas têm pesquisado a respeito da relação entre religião em saúde e sua importância para a saúde física e mental. Muitas pessoas ainda acreditam que divindades podem curar e depositam suas esperanças em crenças religiosas, principalmente os idosos, o que deve ser levado em consideração em uma visão holística do paciente. (MOREIRA-ALMEIDA A, et al., 2006).

É fato que o envelhecimento traz consigo inúmeras alterações fisiológicas, muitas vezes patológicas como doenças crônicas, tal condição deixa os idosos em uma posição de maior vulnerabilidade sendo mais suscetíveis a quadros de depressão (CUNHA JXP, et al., 2012).

Portanto, o profissional de Enfermagem deve ser capaz de identificar as necessidades e problemas relacionados ao quadro depressivo para planejar intervenções que promovam o autocuidado, autonomia e saúde espiritual, aliando quando possível a religiosidade do cliente, garantindo o bem estar e felicidade dos idosos (GARCIA A, et al., 2006).

\section{As Classes e suas descrições:}

\section{Classe 1: Aumento da expectativa de vida frente a comorbidade com os idosos}

A ampliação da comorbidade é um dos fatores relacionados ao crescente número de doenças crônica nãotransmissível, que irão influenciar no aparecimento de doenças psíquicas. A depressão pode estar diretamente relacionada ao estado de saúde física no qual a pessoa se encontra (DUARTE MB e REGO MAV, 2007). 
Dessarte, com o aumento da expectativa de vida da população brasileira, o número de adoecimentos nos idosos cresceu consideravelmente, devido ao aparecimento de comorbidades juntamente com as doenças psíquicas, principalmente a depressão.

\section{Classe 2: A prevalência de sintomas depressivos em idosos residentes em instituições de longa permanência e condomínios.}

Em decorrência desse aumento do número de idosos e da longevidade da população, a que se somam às dificuldades socioeconômicas e culturais que envolvem os idosos e seus familiares e/ou cuidadores, o comprometimento da saúde do idoso e da família, a ausência de cuidador no domicílio e os conflitos familiares, cresce a demanda por Instituições de Longa Permanência para Idosos (ILPI) (GUIMARAES LA, et al., 2019). Entre os idosos que vivem em ILPI, há a prevalência de quadro depressivo em relação àqueles que moram com suas famílias, isso se dá pelo difícil convívio e adaptação.

Os Condomínios para Idosos que tem a proposta de garantir o direito à moradia e promover qualidade de vida para essas pessoas. Apesar da oferta de atividades pela organização desta modalidade habitacional, evidenciou-se que os idosos com depressão são justamente aqueles que, por si só, não participam das atividades. Assim, é importante que os profissionais identifiquem precocemente estes idosos e desenvolvam estratégias de fortalecimento de vínculo, com o objetivo de impedir a evolução do quadro e contribuir para a promoção de sua saúde mental e qualidade de vida (TESTON EF, et al. 2014).

\section{Classe 3: Religiosidade e saúde: atuação ética do enfermeiro}

Constata-se uma variedade de modos por meio dos quais o envolvimento religioso influencia positivamente a vida e a saúde das pessoas (FREITAS MH, 2006). Diante desse estudo, um indivíduo que possui conhecimentos éticos consegue desenvolver em seu âmbito de trabalho mecanismo que ajudem a equipe, e a ele mesmo a cuidar de uma diversidade de pacientes, conciliando a formação ética com a prática médica buscando condutas éticas e morais adequadas a este profissional, na qual a crença desse paciente pode possibilitar uma melhora fisiológica no seu organismo, a qual irá ocasionar um estado de estabilidade psicológica no paciente.

Observou-se que pessoas atribuem a Deus o aparecimento ou a resolução dos problemas de saúde (FARIA JB e SEIDL EMF, 2014). Vale ressaltar que a postura do profissional da saúde diante da diversidade de etnias, culturas e religiões, inclui-se ao ensino da bioética, em que se prepara socialmente o profissional da saúde a obter condutas humanizadas em seu ambiente de trabalho podendo interagir com qualquer indivíduo, na qual ao deparar-se com casos de idosos que por questões de crenças religiosas negam-se a se submeter a consultas que podem comprovar seu diagnóstico de depressão, intervém diretamente nas possíveis intervenções de enfermagem dificultando a atuação do profissional de saúde.

\section{Classe 4: Prevalência de sintomatologia depressiva em idosos.}

O envelhecimento traz mudanças físicas e mentais que requerem adaptações do estilo de vida, como mudanças alimentares e de atividades diárias por conta de limitações ou doenças crônicas características dessa fase. Tais alterações podem acarretar sofrimento psicológico como a depressão, principalmente pela falta de estímulos, promoção da autonomia e do autocuidado, pois ainda é prevalente a ideia de que os idosos não devem realizar suas atividades sem assistência de outrem, o que reforça o sentimento de incapacidade. Portanto é necessário o desenvolvimento de atividades de promoção ao autocuidado e autonomia dessa população visando a melhora da sua qualidade de vida (SANTOS ZMSA, et al. 2012).

Os sintomas depressivos em idosos apresentam alta prevalência na sociedade brasileira. Segundo estudo realizado em 2006, em algumas comunidades essa prevalência varia entre 13,0 a 38,0\%. A sintomatologia depressiva em idosos está relacionada a diversos fatores como diminuição da qualidade de vida, aparecimento de doenças físicas comuns no Envelhecimento, dependência para a realização de atividades diárias, condição econômica precária e abandono familiar. Além disso nota-se também, a maior frequência de sintomas depressivos no sexo feminino, ocasionado pela maior vulnerabilidade social (MENDES-CHILOFF CL, et al., 2018). 


\section{A correlação entre classes}

Ainda que a expectativa de vida tenha chegado em 74,8 anos em 2013, uma parte da população idosa ainda apresenta várias comorbidades, devido existirem poucas políticas públicas acerca da prevenção de doenças crônicas juntamente com doenças psíquicas (FERNANDEZ JCA, 2014).

Além disso, a cultura é uma grande influenciadora na expectativa de vida e também no surgimento de doenças, pois muitas pessoas deixam de buscar ajuda por conta do etnocentrismo enfrentando, pois poucos profissionais adotam práticas de cuidado cultural que sejam congruentes com as crenças e padrões de comportamento (CAMARGOS MCS e GONZAGA MR, 2015).

Estudos realizados indicam que a religiosidade está relacionada à melhoria da qualidade de vida, influenciando positivamente, inclusive, os que estão acometidos por alguma doença. Dessa forma, idosos com comorbidades podem ter uma recuperação mais rápida de seu estado de saúde se motivados a mobilizarem a energia positiva a qual implica a religiosidade, a fé, pois as pessoas que possuem fé sentemse mais fortes para enfrentar dificuldades e continuar a lutar pela sua sobrevivência, acreditando que serão curadas dos seus males (MURAKAMI R e CAMPOS CJG, 2012).

Porém, mesmo com sua grande importância, a religiosidade ainda é pouco abordada e o profissional de saúde tem dificuldades para lidar com questões religiosas para motivar seus pacientes, uma vez que essa temática não faz parte de sua formação. Assim, analisa-se a necessidade de implantar a espiritualidade e a religiosidade na graduação desse profissional (INOUE TM e VECINA MVAV, 2017).

Os idosos com sintomas de depressão negligenciados quanto ao seu diagnóstico e tratamento, possuem qualidade de vida alterada. O enfermeiro possui um papel importante na observação constante desses idosos, a fim de proporcionar um diagnóstico de depressão precoce, visando um tratamento adequado, com o intuito de os incluir novamente em suas relações sociais (SILVA ER, et al, 2012). Ademais, o cuidado individualizado reconhece a singularidade e os valores dos pacientes considerando suas características pessoais e suas condições clínicas (MARTINS PF e PERROCA MG, 2017).

\section{CONSIDERAÇÕES FINAIS}

Um tópico que requer análise sensata e urgente consiste sobre a atuação do enfermeiro frente aos processos de cuidado com os pacientes idosos que apresentam quadro depressivo, em que se foi analisado aspectos de vulnerabilidade devido o abandono familiar e social. Evidenciando a necessidade do desenvolvimento de pesquisa sobre estes temas visto o atual crescimento da população idosa e dos quadros de transtornos psicológicos. O profissional deve orientar o paciente a desenvolver mecanismo que possibilitem o autocuidado, podendo acarretar em uma autoconfiança e sentimento de autonomia. Quanto às intervenções de enfermagem, nota-se que o profissional desta área possui um papel fundamental no tratamento do paciente idoso acometido de depressão.

\section{REFERÊNCIAS}

1. BEZERRA FC, et al. Estudos sobre envelhecimento no Brasil: revisão bibliográfica. Rev. bras. geriatr. gerontol., 2012; 15(1): 155-167.

2. CAMARGO BV, JUSTO AM. IRAMUTEQ: um software gratuito para análise de dados textuais. Temas psicol., 2013. 21(2): 513-518.

3. CAMARGOS MCS, GONZAGA MR. Viver mais e melhor? Estimativas de expectativa de vida saudável para a população brasileira. Cad. Saúde Pública, 2015; 31(7): 1460-1472

4. CARMO EH, et al. Mudanças nos padrões de morbimortalidade da população brasileira: os desafios para um novo século. Epidemiol. Serv. Saúde, 2013; 12(2): 63-75.

5. CORREA ERP, MIRANDA-RIBEIRO A. Ganhos em expectativa de vida ao nascer no Brasil nos anos 2000: impacto das variações da mortalidade por idade e causas de morte. Ciência \& Saúde Coletiva, 2017; 22(3).

6. CUNHA JXP, et al. Autonomia do idoso e suas implicações éticas na assistência de enfermagem. Saúde debate, 2014; 36(95): 657-664. 
7. DUARTE MB, REGO MAV. Comorbidade entre depressão e doenças clínicas em um ambulatório de geriatria. Cad. Saúde Pública, 2007; 23(3):691-700.

8. EVANGELISTA RA, et al. Percepções e Vivências dos Idosos Residentes de uma Instituição Asilar. Rev. esc. Enferm., 2014; 48(2): 81-86.

9. FARIA JB, SEIDL EMF. Religiosidade, enfrentamento e bem-estar subjetivo em pessoas vivendo com HIV/aids. Psicol. estud. 2006; 11(1): 155-164.

10. FERNANDEZ JCA. Determinantes culturais da saúde: uma abordagem para a promoção de equidade. Saúde e Sociedade, 2014; 23(1).

11. FLORINDO S, et al. Depressão no idoso: Diagnóstico, Tratamento e Benefícios da Atividade Física, 2002; 8(3): 9198.

12. FREITAS MH. Religiosidade e saúde: experiências dos pacientes e percepções dos profissionais Revista Pistis \& Praxis: Teologia e Pastoral, 2014; 6(1): 89-10.

13. GARCIA A, et al. A depressão e o processo de envelhecimento. Ciênc. cogn., 2006; 7(1): 111-121.

14. GUIMARAES LA, et al. Sintomas depressivos e fatores associados em idosos residentes em instituição de longa permanência. Ciênc. saúde coletiva, 2019; 24(9): 3275-3282.

15. INOUE TM, VECINA MVAV. Espiritualidade e/ou religiosidade e saúde: uma revisão de literatura. J Health Sci Inst., $2017 ; 35(2): 127-30$

16. LOBO AJS, et al. Nível de dependência e qualidade de vida da população idosa. Rev. bras. enferm., 2014; 67(6): 913-919.

17. MARTINS PF, et al. Necessidades de cuidados: o olhar do paciente e a equipe de enfermagem. Rev. Bras. Enferm., 2017; 70(5): 1026-1032.

18. MENDES KDS, et al. Revisão integrativa: método de pesquisa para a incorporação de evidências na saúde e na enfermagem. Texto contexto - enferm., 2008; 17(4): 758-764.

19. MENDES-CHILOFF CL, et al. Sintomas depressivos em idosos do município de São Paulo, Brasil: prevalência e fatores associados (Estudo SABE). Rev. bras. epidemiol., 2018; 21(2): 180014.

20. MOREIRA-ALMEIDA A, et al. Religiosidade e saúde mental: uma revisão. Rev. Bras. Psiquiatr, 2006; 28(3): $242-250$.

21. MURAKAMI R, CAMPOS CJG. Religião e saúde mental: desafio de integrar a religiosidade ao cuidado com o paciente. Rev. bras. enferm., 2012; 65(2): 361-367.

22. SANTOS ZMSA, et al. Autocuidado universal praticado por idosos em uma instituição de longa permanência. Rev. bras. geriatr. gerontol., 2012; 15(4): 747-754.

23. SILVA EP, et al. Perceptions of care among elderly couples. Rev. bras. geriatr. gerontol., 2019; 22(1): $180-136$.

24. SILVA PLN, et al. Psychological and nursing assistance to the elderly in primary care. Revista de Pesquisa Cuidado é Fundamental Online, 2014, 6(4):1707-1718.

25. SILVA ER, et al. Prevalência e fatores associados à depressão entre idosos institucionalizados: subsídio ao cuidado de enfermagem. Rev. esc. enferm., 2012; 46(6); 1387-1393.

26. TESTON EF, et al. Sintomas depressivos em idosos: comparação entre residentes em condomínio específico para idoso e na comunidade. Revista Brasileira de Enfermagem, 2014; 67(3): 450-456. 